Bull. Mater. Sci., Vol. 3, Number 2, July 1981, pp.

\title{
Critical point phenomena, heat capacities and the renormalization group theory of fluctuations
}

\author{
E S R GOPAL \\ Department of Physics, Indian Institute of Science, Bangalore 560 012, India
}

MS received 23 January 1981

\begin{abstract}
In the normal study of matter, the ordered state is considered first, followed by the addition of minor disorder or fluctuations, for instance, studying crystalline solids with some quasiparticle excitations like phonons and magnons. The discovery of the universality of critical point phenomena seems to provide a chance to study a regime dominated by the fluctuations. The Onsager solution of the two-dimensional Ising model, exhibiting a logarithmic singularity in heat capacity, and the FairbankBuckingham-Kellers experiments, showing such a singularity in the heat capacity near the superfluid transition of liquid $4 \mathrm{He}$, are landmarks in this topic. The recent renormalization group theory shows a way of studying the patterns among the fluctuations. The dependence of the critical exponents upon spatial and spin dimensionalities, the existence of universal amplitude ratios and the other aspects of critical phenomena are briefly discussed.
\end{abstract}

Keywords. Critical phenomena; specific heats; fluctuations; renormalization group theory.

\section{Introduction}

The theories of materials start usually with a perfect crystalline arrangement of the particles. This regular arrangement is then disturbed by situations like the thermal vibrations of the particles and the presence of vacancies or dislocations, so that one can study the behaviour of real materials by starting from the framework of a perfectly ordered solid. In order to make the study feasible, one also assumes that the defects or disturbances are small and that they alter the basic structure only in a minor way. The situation is nearly the same when one considers the general framework of say the quantum theory. The ground state is the starting point and one calculates the effects of perturbations upon the ground state Hamiltonian. The calculation is simple if the disturbance is weak. The perturbation theory is successful for the cases of weak disturbances or small fluctuations.

Thus in most situations, one starts with an ordered state and then studies the effects of a small perturbation or disorder. The study is feasible and successful only if the perturbations or fluctuations are small. One does not want a case in 
which the fluctuations are large and indeed one does not even know how to handle a case in which the fluctuations and perturbations are large.

In the last few years, it appears that one has found a way to handle some fluctuation-dominated situations so that one is beginning to see patterns even in chaos and disorder. These patterns or symmetries are naturally of a special kind. However concepts like invariance or scaling are applicable as equally to the new situations as to the more traditional older examples.

\section{Critical point phenomena: A fuctuation-dominated region}

In order to study the patterns and properties of fluctuations, one must first of all look for the phenomena in which they are dominant. A simple calculation in statistical mechanics (Hill 1960; Morse 1969; Gopal 1974) shows that the mean square value of the fluctuations in say the density $\rho$ is given by

$$
\overline{(\delta \rho)^{2}}=\rho^{2} k T \chi_{T} / V,
$$

where $V$ is the volume of the sample at a temperature $T$ and $X_{T}=-V^{-1}(\partial V / \partial P)_{T}$ is the isothermal compressibility. Under ordinary conditions, the fluctuations are of the order of $\left(\overline{\delta \rho)^{2}} / \rho^{2} \approx 1 / N\right.$ where $N$ is the number of particles in the system. Since $N \approx 10^{28} / \mathrm{m}^{3}$, the fluctuations are completely negligible. Thus the idea of starting with the average or ground state values and treating the fluctuations as small perturbations works very well.

A situation in which the compressibility $X_{T}$ becomes very large is indeed known for more than a hundred years. The observation of the critical temperature of a gas like carbondioxide by Andrews and the development of the Van der Waals equation of state, providing the continuity of the liquid and the gas states, have become elementary textbook topics. The isotherms of a gas at temperatures $T$ much greater than the critical temperature has a simple hyperbolic shape (figure 1a). At temperatures below $T_{c}$, the matter is in the liquid phase at high densities, in a
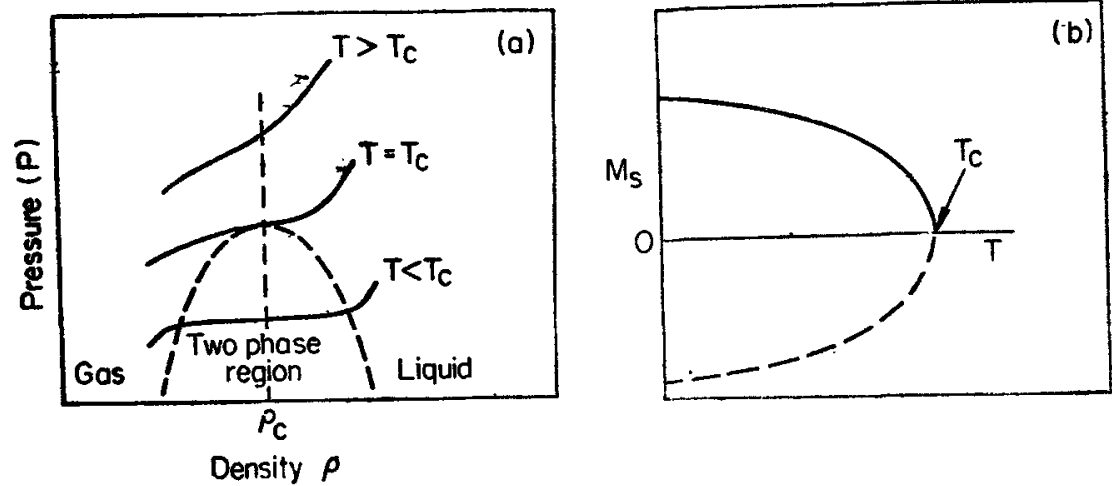

Figure 1. (a) Isotherms of a liquid + gas system in the critical region. The coexistence curve boundary is shown by a broken line. (b) Spontaneous magnetization $M_{s}$ of a ferromagnet near the Curie temperature. Note the broken line showing up or down magnetization so that a rotation of the curve by $90^{\circ}$ makes it similar to the coexistence curve of a liquid + gas system. 
vapour phase at low densities and in a coexisting phase at intermediate densities. At the critical temperature $T_{c}$, the compressibility $\rho^{-1}(\delta \rho / \delta P)_{T}$ becomes infinitely large and from equation (1), one should expect very large fluctuations. The manifestation of these large fluctuations in the form of critical opalescence - (scattering of the light falling on the system tosuch an extent that the medium appears to glimmer or opalesce) - was known at the turn of the last century. The explanation of the phenomenon of opalescence as the scattering of light from the density fluctuations was advanced by Einstein and by Smoluchowski about seventy years ago. Since that time light scattering has become a convenient tool to study the fluctuations in a system (Benedek 1968; Gopal 1980).

The unusual opportunity presented by a large increase in fluctuations was recognized only in recent times. In the fifties and sixties, when careful measurements in the critical region were performed, certain interesting features became clear. For example when the density difference $\rho_{L}-\rho_{G}$ between the coexisting liquid and gas phases was expressed as a function of the temperature difference $T_{c}-T$ near the critical temperature, the function assumed a simple power law form

$$
\left(\rho_{L}-\rho_{G}\right)=B\left(T_{C}-T\right)^{\beta}
$$

with $\beta \approx 0.34$ for a number of gases. Similarly the specific heat at constant volume became very large as

$$
C_{V} \sim\left|T_{c}-T\right|^{-a}
$$

with $\alpha \approx 0.1$, where the symbolic way of writing (3) denotes the asymptotic functional form as $\left|T-T_{c}\right| \rightarrow 0$. The compressibility of the fluid in the critical region had a divergence

$$
X_{T} \sim\left|T-T_{c}\right|-\gamma
$$

with $\gamma \approx 1.3$. Other properties also showed such simple power law behaviour.

The situation became even more interesting when the properties of ferromagnets were studied near the Curie temperature. It is known, for example, that the simple thermodynamic properties of magnetic systems are formally obtained by the equivalence $M+\rho, H \leftrightarrow P$ in the equations. Thus one can calculate the fluctuations in magnetization using the formal analog of equation (1). When the specific heats of ferromagnets were measured near $T_{c}$, it was found that

$$
C_{M} \sim\left|T-T_{c}\right|^{-a},
$$

with the simple power law exponent $\alpha$ having the same value $\approx 0.1$ as with (3). The spontaneous magnetization $M_{s}$ went to zero at $T_{c}$ with a functional form

$$
M_{s} \sim\left(T_{c}-T\right)^{\beta},
$$

with $\beta$ having the same value $\approx 0.34$ as in (2). The magnetic susceptibility, which is the analog of the compressibility had the same value 1.3 for the exponent $\gamma$. In 
other words the critical exponents appeared universal, independent of the chemical nature of the interparticle forces, Van der Walls type or hydrogen-bonded type or exchange interaction or any other type (figure 2). This universality or the independence from the type of interatomic forces immediately suggested that the behaviour must be the result of very general causes, the results of large scale fluctuations dominating over the features specific to magnetic interactions or cohesive forces.

More detailed studies revealed yet another facet of the critical point phenomena, namely, the scaling of the properties. A property like the specific heat, when plotted in terms of reduced variables $C / N R$ against $T / T_{c}$, gave the same curve for all the substances. Thus by suitable choice of the reduced variables, a property of all the substances fell on a single curve, depending of course on this particular property (Stanley 1971; Green 1971).

While the universality feature showed that the critical point phenomena had the origins in the large scale fluctuations, it also became clear that these fluctuations must have some pattern or symmetry or structure to account for the simple power law and the scaling behaviour of the properties.
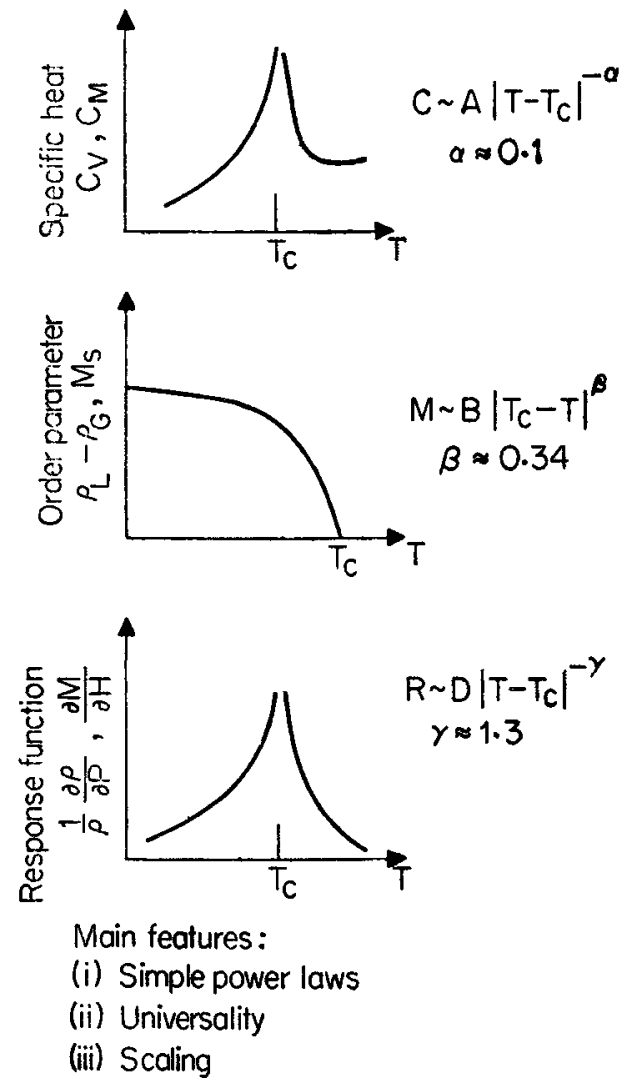

Figure 2. Universality of critical phenomena. The heat capacity, the order parameter and the response function show the same power law exponents for liquid + gas systems, ferromagnets, antiferromagnets, order-disorder transitions of alloys near their critical temperature. 


\section{Behaviour of heat capacity}

The behaviour of heat capacity has a special place in the study of critical phenomena. A simple model of a system showing a cooperative transition is the so-called Ising model. Here spins $\vec{S}_{i}$ are placed on the lattice sites and interact only with nearest neighbours with a Hamiltonian

$$
H_{I}=-2 J \vec{S}_{i z} \cdot \vec{S}_{j z}
$$

In other words only the $z$-component of the interaction term is retained in the full Heisenberg exchange interaction

$$
H_{H}=2 J \overrightarrow{S_{i}} \cdot \vec{S}_{j}
$$

For spin $\frac{1}{2}$ particles, the Ising interaction has only two values, adjacent spins parallel or antiparallel. Such a model is a simplification of the situation in a ferromagnet. The order-disorder transition, in which adjacent sites can be occupied by correct or incorrect atom pairs, and a liquid +gas model, in which a neighbouring site may be occupied or vacant, are the other situations which can be approximated by the Ising model.

The approximate treatment of the Ising model by Bragg-Williams, Bethe and others had indicated a phase transition in which the heat capacity was finite but discontinuous across the transition, a situation called a thermodynamic second-order phase transition. The subject took a new turn in 1944 when Onsager managed to solve the two-dimensional zero magnctic field case exactly (Onsager 1944). This statistical mechanical solution is even today a remarkable feat of mathematical analysis and even the so-called greatly simplified versions of the analysis are far beyond the understanding capability of average physicists and chemists (McCoy and Wu 1973). The Onsager solution showed that the two-dimensional Ising model had a symmetrical logarithmic infinity in heat capacity at $T_{c}$. Thus

$$
C_{\mathrm{H}=0} \approx A \ln \left|T-T_{c}\right|+\ldots
$$

where the other terms are nonsingular terms small in comparison. The simplicity of the model and the unexpected behaviour of the solution has prompted many investigations of the full three-dimensional Ising model. Unfortunately even today it has not been solved exactly and remains one of the foremost challenges in mathematical physics.

The experimental study of the heat capacity near the critical temperature took a great leap forward when in 1956 Buckingham, Fairbank and Kellers studied liquid ${ }^{4} \mathrm{He}$ near its lambda-transition at $2 \cdot 16 \mathrm{~K}$ (Buckingham and Fairbank 1961). The temperature could be controlled to a microdegree and over an extended range of temperature the specific heat fitted the singularity

$$
C_{p}=A_{ \pm}+B \ln \left|T-T_{\lambda}\right|,
$$




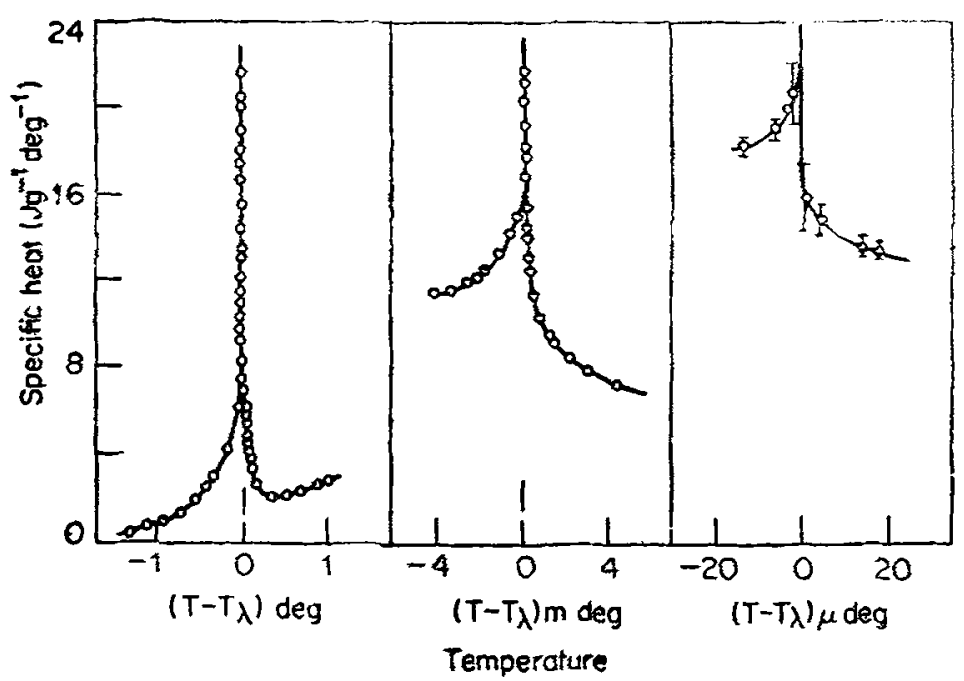

Figure 3. Heat capacity of liquid 'He near the $\lambda$-transition, based on the experiments of Buckingham, Fairbank and Kellers. The portion shown by the thin vertical line in the $(a)$ portion with $\mathrm{X}$-axis in degrees, is expanded to the $(b)$ portion with the $X$-axis in millidegrees. The vertical line in this figure is expanded to the (c) portion with the $X$-axis in microdegrees. The broken line is the theoretical fit to the data.

as shown in figure 3. More recent investigations have gone to $\left|T-T_{\lambda}\right| \approx 10^{-8} \mathrm{~K}$ and a functional form

$$
C_{p} \sim D_{ \pm}+E_{ \pm}\left|T-T_{\lambda}\right|^{-0.02},
$$

seems to fit the data better (Ahlers 1980). For practical purposes the two forms are indistinguishable since

$$
\operatorname{Lt}_{a \rightarrow 0}\left[\left(t^{-a}-1\right) / \alpha\right]=\ln |t| \text {. }
$$

Since these pioncering experiments on liquid ${ }^{4} \mathrm{He}$, the topic of specific heats near phase transitions has become an important and fruitful area of work (Kumar et al 1981).

\section{Renormalization group calculation of critical exponents and anplitudes}

An understanding of the role of fluctuations in determining the critical phenomena has come in the last 10 years due to the ingenious application of the renormalization group theory by Wilson and others (Wilson and Kogut 1974, Ma 1976). As mentioned earlier the interatomic length scale becomes irrelevant when the correlation lengths become very large. The scale invariance is treated in an ingenious way to tackle the problem involving the fluctuations of about $10^{23}$ particles. The shortest wavelength fluctuations are eliminated in such a way that the resulting problem, involving a fewer degrees of freedom, looks similar in form to the original 

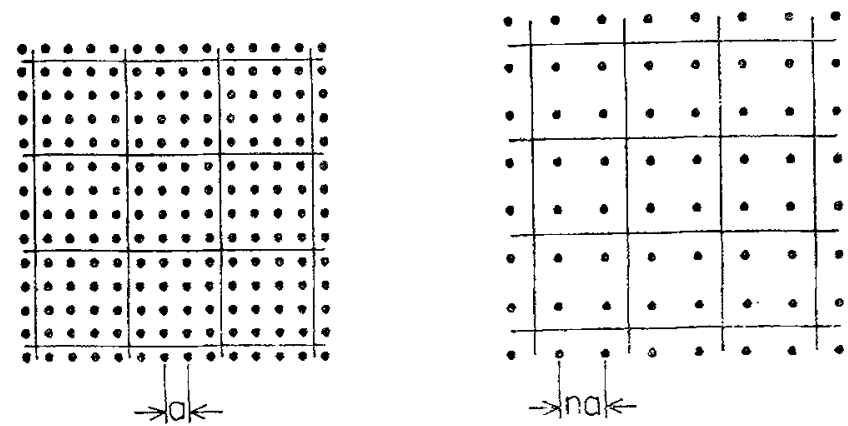

Figure 4. Concept of scale invariance. The properties of a block of large number of spins are similar to the scaled properties of a block of smaller number of spins, being dependent only on the scaling factor $\mathbf{n}$ and not on the interatomic scale of length $\mathbf{a}$.

one but with a different set of coupling constants. This process is repeated until finally there is left a problem with just one degree of freedom whose features could be studied by more conventional methods. In other words, the initial Hamiltonian is transformed into a sequence of effective Hamiltonians by successively eliminating the fluctuations starting with one having shortest wavelength. The complicated $N$ particle ( $N$ of the order of $10^{23}$ ) problem in a known simple Hamiltonian is transformed into a one-particle problem with a transformed Hamiltonian (compare figure 4). These transformations belong to a mathematical group called the renormalization group.

Under the repeated transformations, the Hamiltonian moves along a line in the configuration space. After the small scale fluctuations are integrated out, the Hamiltonian does not move and is said to have reached a fixed point of the transformation. This is so because the correlation length is infinite at $T_{c}$ and will remain so for finite changes of scale. The eigenvalues of the transformation about the fixed point give the critical exponents. Thus the exponents come out to be independent of the initial couplings and depend only upon the universal properties of the fixed point. Any such mathematical technique is productive only if the final results are tractable. Wilson showed that this is indeed so and calculated the exponents as an asymptotic series.

Unfortunately even the simplest details of the renormalization group theory (Ma 1976; Maris and Kadanoff 1978) are still more complex than what the average experimental scientist can follow. Under these circumstances it is better to quote the results than attempt an explanation. The RG theory shows that the critical exponents depend upon the spatial dimensionality $d$ of the system in a major way and upon the spin dimensionality $n$ in a minor way. The Ising system (equation 7) has $n=1$ since only the $Z$ component is allowed. The Heisenberg interaction has $n=3$; liquid helium- 4 in its superfluid phase has a complex wave function and belongs to the $n=2$ category. The specific heat exponent $\alpha$ is given by an equation

$$
\alpha=-\frac{(n-4)}{2(n+8)}(4-d)-\frac{n^{3}+32 n^{2}+116 n+112}{4(n+8)^{3}}(4-d)^{2}+\ldots
$$


For the three dimensional case $d=3$, the values are $-0.10,-0.02$ and +0.08 for $n=3,2,1$ respectively. Note that a positive value of $\alpha$ means an infinitely large value of the specific heat at $T_{c}$, while a negative value means a cusp at $T_{c}$.

The best experimental value of the exponent $\alpha$ in the case of liquid helium- 4 near its superfluid transition was already mentioned as $-0.02 \pm 0.01$ and this compares very well with the theoretical value for $d=3, n=2$ mentioned above. The $d=3, n=1$ systems are the Ising magnets, liquid + gas critical systems and binary liquids near their critical solution temperature. The recent experimental values cluster round $0.10 \pm 0.04$ which is again in good agreement. The Heisenberg magnet systems have also been studied carefully and yield $\alpha=-0.12 \pm 0.05$. Thus one has remarkable explanation of the experiments.

The RG theory is able to further study the pattern of fuctuations and the correlations among them. In particular if the singular part of the specific heat above $T_{c}$ and below $T_{c}$ are written $a^{\circ}$

$$
\begin{aligned}
C_{V} & =A^{+}\left|T-T_{c}\right|^{-a} \text { for } T>T_{c}, \\
& =A^{-}\left|T_{c}-T\right|^{-a^{\prime}} \text { for } T<T_{c},
\end{aligned}
$$

the RG theory shows that

$$
\begin{aligned}
& \alpha=\alpha^{\prime} \text { and } \\
& A^{+} / A^{-}=2^{\alpha} \cdot \frac{n}{4}\left[1+(4-d)+(4-d)^{2}\left\{\frac{3 n^{4}+74 n^{3}+708 n^{2}+2264 n+6400}{2(n+8)^{4}}\right.\right. \\
& \left.\left.+\frac{4-n}{2(n+8)} \zeta(2)-\frac{3(5 n+22)}{(n+8)^{2}} \zeta(3)+\frac{9(4-n)}{4(n+8)^{3}}(-2 \cdot 349)\right\} \ldots\right]
\end{aligned}
$$

In other words the amplitudes of the anomalies above and below $T_{c}$ bear a universal ratio. For $d=3$, the calculated values are $1.24,0.88$ and 0.48 for $n=3,2,1$ respectively. The experimental values are respectively $1.3 \pm 0.1,0.9 \pm 0.1,0.5 \pm 0.1$ for the three cases, again showing broad agreement (Kumar et al 1981).

In addition to these patterns among the fluctuations, yielding the values of the exponents and the amplitude ratios, the RG theory goes further to calculate the finer details also. For example, it is shown that simple equations like (2), (3), (4), (5), (6), (13) are valid only in the asymptotic limit of $\left|T-T_{c}\right| \rightarrow 0$. For slightly larger values of $\left|T-T_{c}\right|$, one can write the higher order term of equation (13) as

$$
C_{V}=A\left|T-T_{c}\right|^{-a}+B\left|T-T_{c}\right|^{-a+\Delta}+\ldots
$$

and $\Delta$ the correction to asymptotic exponent has a universal value of nearly 0.5 . One can get the ratio of the correction amplitudes above and below $T_{c}$. These universal values arise because of the presence of well-defined patterns among the fluctuations.

The fluctuations also show spatial and temporal correlations as $T_{c}$ is approached. These correlations can be studied using methods like the laser light scattering. 
Certain averages of the correlations are related to the transport coefficients likc viscosity, diffusion or electrical resistivity and these properties are also the objects of intensive study in the last few years (Hohenberg and Halperin 1977; Ginzburg et al 1980).

\section{Acknowledgement}

The author would like to thank his many colleagues over many years for many useful discussions. Over these years our research has also been supported by project funds from the UGC, the CSIR, the DAE and the DST as well as the general support from the Institute and he would like to express his thanks to these agencies also.

\section{$\mathbf{R} \cdot$ ferences}

Ahlers G 1980 Rev. Mod. Phys. 52581

Aenedek G B 1968 Statistical Physics, Phase transitions and superfluidity (eds) M Chretian, E P Gross and S Deser (New York: Gordon and Breach) Vol. 2 p. 1

Buckingham M J and Fairbank W M 1961 Prog. Low Temp. Phys. 380

Ginzburg V L, Levenyuk A P and Sobyanin A A 1980 Phys. Rep. 57151

Gopal E S R 1974 Statistical mechanics and properties of matter (New York : John Wiley) (1978 New Delhi: McMillans)

Gopal E S R 1980 in Gravitation, quanta and the universe (eds) A R Prasanna, J V Narlikar and C V Visweswara) (New Delhi : Wiley Eastern)

Green H S 1971 Critical Phenomena (New York: Academic Press Enrico Fermi School)

Hill T L 1960 Introduction to statistical thermodynamics (New York: Addison Wesley)

Hohenberg P C and Halperin B I 1977 Rev. Mod. Phys. 49435

Kumar A, Krishnamurthy H R and Gopal E S R 1981 Equilibrium critical phenomena in binary liquid systems, NTPP Report (to be published).

Ma S K 1976 Modern theory of critical phenomena (Reading: Benjamin)

Maris H J and Kadanoff L P 1978 Am. J. Phys. 46652

McCoy B and Wu T T 1973 Two-dimensional Ising model (Cambridge Mass: Harvard Univ. Press)

Morse P M 1969 Thermal physics (New York: Benjamin)

Onsager L 1944 Phys. Rev. 65117

Stanley H E 1971 Introduction to phase transitions and critical phenomena (Oxford: Oxford Univ. Press)

Wilson K G and Kogut J 1974 Phys. Rep. C12 75 